\title{
Electronic and structural properties of rare earth pnictides
}

\author{
RAMAKANT BHARDWAJ* \\ Department of Basic Science, Technocrats Institute of Technology, Bhopal (MP) 462023, India
}

\begin{abstract}
In the present paper structural and electronic properties of rare earth pnictides have been presented. The present calculation has been performed using self-consistent tight binding linear muffin tin orbital (TB-LMTO) method within the local density approximation (LDA). The studied compounds undergo a structural phase transition from NaCl-type structure to CsCl-type structure. The electronic band structure and density of states of the pnictides have been reported. The equilibrium lattice parameter a $(\AA)$, bulk modulus B (GPa), number of f-states at the Fermi level $\mathrm{N}_{\mathrm{f}}$ (states/Ry cell) and volume collapse of AmBi and $\mathrm{CmBi}$ have also been reported. The calculated equilibrium structural parameters are in good agreement with the available experimental results.
\end{abstract}

Keywords: electronic band structure; electronic properties; phase transition; density of states

(C) Wroclaw University of Technology.

\section{Introduction}

The rare-earths become more and more important due to their intricate structural, magnetic, electronic and transport properties. They also have interesting semiconducting properties and are used in a wide range of practical applications in the field of nonlinear optics, electro-optic components, grinding alloys, composite lasers and phosphors lasers [1-8]. Among these rare earth pnictides the rare earth bismuthides contain a pnictogen ion with the largest atomic radius and for this reason, they have been studied to elucidate the role of $\mathrm{p}-\mathrm{f}$ mixing on the structural properties of these compounds. The rare-earths have different occupation numbers for the shallow inner $4 \mathrm{f}$ shell. Due to the unfilled $4 \mathrm{f}$ shells of rare-earth atoms, it is a challenging problem to obtain an accurate theoretical description of the electronic structure of the rareearth compounds. Because of the highly localized nature of the $4 \mathrm{f}$ electrons, direct $\mathrm{f}-\mathrm{f}$ interactions between neighboring rare-earth atoms are generally considered to be nearly negligible [9]. The present pnictides ( $\mathrm{XBi} ; \mathrm{X}=\mathrm{Am}, \mathrm{Cf}$ ) exhibit sixfold coordinated NaCl-type (B1) structure and under high pressure they show a first order structural

*E-mail: drrkbhardwaj100@gmail.com phase transition to CsCl-type (B2) structure at a normal temperature and pressure. Only some theoretical papers and a little experimental work have been dedicated to the study of structural and electronic properties of this series of rare earth pnictides. Therefore, the knowledge of high pressure behavior is important in understanding the properties of these compounds.

Some structural and elastic properties of (XBi; $\mathrm{X}=\mathrm{Am}, \mathrm{Cf})$ were studied in previous years $[10$, 11]. The structural phase transition pressure from B1 phase to body centered tetragonal (BCT) phase at $15 \mathrm{GPa}$ with $12 \%$ volume collapse for $\mathrm{AmBi}$, and B1-B2 phase at 11 to $12 \mathrm{GPa}$ with $7 \%$ volume collapse for CfBi have been reported by Benedict et al. [10]. Ahirwar et al. [11] investigated the pressure induced structural phase transition, elastic and thermal properties of actinide monobismuthides AmBi and CfBi by using a modified interionic potential theory. Recently, the pressure induced $\mathrm{NaCl}$ (B1) to $\mathrm{CsCl}$ (B2) phase transition of actinide pnictides (XBi) (AmBi and $\mathrm{CfBi}$ ) has also been reported in a study of interaction potential model (MIPM) (including the covalency effect) [12]. To the best of our knowledge no information about the electronic properties of rare earth pnictides has been reported in the literature. Therefore, it is interesting to study the electronic 
properties of the pnictides using the first principle tight binding linear muffin tin orbital (TB-LMTO) method.

In the present paper, the ground state electronic structure and structural properties of rare earth pnictides of NaCl-type and CsCl-type structures have been studied by first principle tight binding linear muffin tin orbital (TB-LMTO) method within the local density approximation (LDA). In the present study it has been found that these solids crystallize in NaCl-type structure. They undergo a first order phase transition from NaCl-type to CsCl-type structure in the pressure range of 11.8 to 14.7 GPa. The electronic band structure (BS) and density of states (DOS) of these pnictides are also reported. The rest of the paper is organized as follows: Section 2 describes the method of calculation of electronic band structure (BS) and phase transition pressure. In Section 3 some interesting results have been discussed. Finally, in Section 4 the conclusions of the present study are presented.

\section{Method of calculation}

The rare earth pnictides considered in this work crystallize in NaCl-type (Fm3m, 225) structure at ambient conditions. The rare earth atom is positioned at $(0,0,0)$ and bismuth at $(1 / 2,1 / 2,1 / 2)$. The studied rare earth pnictides have a CsCl-type (Pm3m, 221) structure in which bismuth is positioned at $(1 / 2,1 / 2,1 / 2)$. For loosely packed structures, empty spheres are introduced in appropriate amounts into $\mathrm{NaCl}$ phase without breaking the crystal symmetry. The TB-LMTO method works well for the close packed structures, however, the present compounds belong to $\mathrm{NaCl}$ phase, which is not close packed at ambient conditions. Therefore, for these loosely packed structures two equivalent empty spheres at positions $(0.25,0.25,0.25)$ and $(0.75,0.75,0.75)$ have been introduced in such way that they do not break the crystal symmetry [13]. For $\mathrm{CsCl}$ structure these empty spheres are not needed because it is a close packed structure. The electronic band structures have been calculated using the self-consistent tight binding linear muffin tin orbital (TB-LMTO) method $[14,15]$ within the local density approximation (LDA) [16]. Van Barth and Hedin [17] parameterization scheme was used for the exchange correlation potential. To minimize the errors in the LMTO method, combined correction terms were included. These terms account for the non-spherical shape of the atomic spheres and the truncation of the higher partial waves inside the sphere. Between the atoms, the charge flows according to the electronegativity criteria [18]. The partial waves of $\mathrm{s} p \mathrm{~d}$ and $\mathrm{f}$ states were taken into account. The $\mathrm{E}$ and $\mathrm{k}$ convergences were checked subsequently to achieve better accuracy. The calculations were performed for $512 \mathrm{k}$ points (the grid of $8 \times 8 \times 8$ ) in the Brillouin zone for both the B1 and B2 phases. To obtain the total energy and partial density of states the tetrahedron method of Brillouin zone integration was used [19]. The total energy was computed by reducing the volume from $1.05 \mathrm{~V}_{0}$ to $0.60 \mathrm{~V}_{0}$, where $\mathrm{V}_{0}$ is an equilibrium cell volume. The calculated total energy was fitted to Birch equation of state [20] to obtain the pressure-volume relation. The pressure was obtained by taking the volume derivative of total energy. The bulk modulus $\left(\mathrm{B}=-\mathrm{V}_{0} \mathrm{dP} / \mathrm{dV}\right.$ ) was also calculated from the P-V relation. The stability of a particular structure was decided by the minima of the enthalpy. The phase transition pressure can be obtained by matching the enthalpies of both structures such that the difference of enthalpy $\Delta \mathrm{H}=\mathrm{H}_{\mathrm{B} 2}-\mathrm{H}_{\mathrm{B} 1}$ becomes zero at transition pressure.

\section{Result and discussion}

\subsection{Structural properties}

The electronic band structures BS calculations were performed to estimate the total energy of rare earth pnictides by using the first principle TBLMTO method. The total energies for the pnictides have been plotted against different compressions in Fig. 1 and Fig. 2. The minimum of all curves defines the equilibrium volume $\mathrm{V}_{0}$ (or equilibrium $\mathrm{a}_{0}$ ), which has been found to be $57.5 \AA^{3}$ for $\mathrm{AmBi}$ whereas the corresponding parameter a of $6.09 \AA$ is underestimated by $11.2 \%$. The same calculations performed for $\mathrm{CfBi}$ estimated the equilibrium 


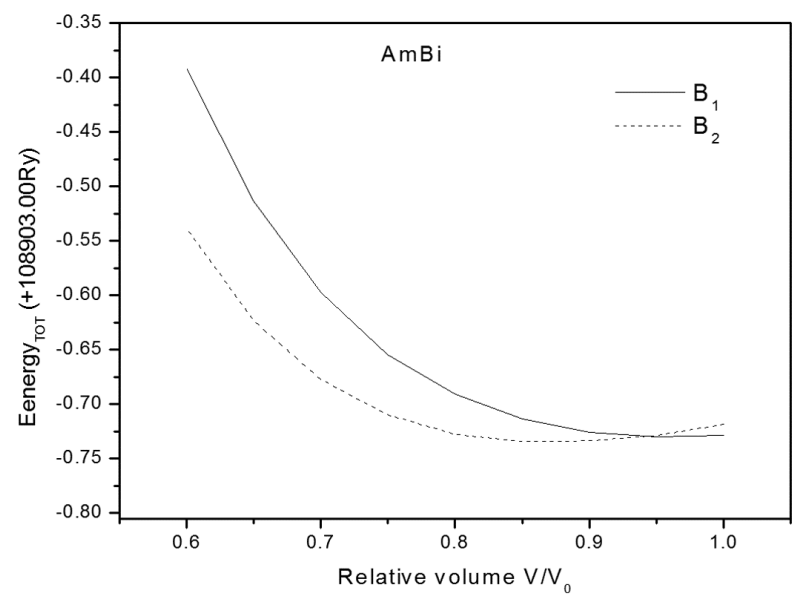

Fig. 1. Variation of total energy with relative volume of $\mathrm{NaCl}$ and $\mathrm{CsCl}$ phases for $\mathrm{AmBi}$.

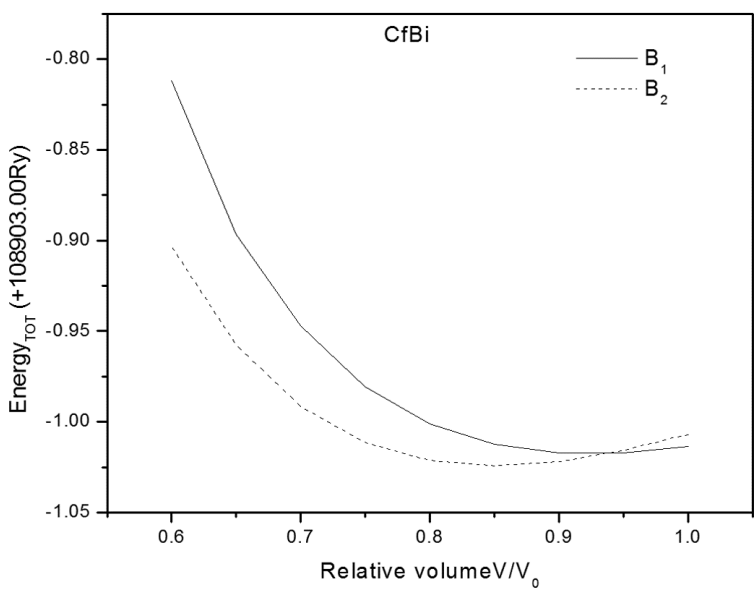

Fig. 2. Variation of total energy with relative volume of $\mathrm{NaCl}$ and $\mathrm{CsCl}$ phases for $\mathrm{CfBi}$.

volume as $55.2 \AA^{3}$ and the corresponding parameter $\mathrm{a}=6.01 \AA$, underestimated by $6.5 \%$. These values have been compared with experimental $[8,10]$ and theoretical $[11,12]$ data. It is clear from Table 1 that our calculated values are in better agreement with the experimental $[8,10]$ than the other theoretical results [11, 12].

The calculation of phase transition pressure was carried out by estimating enthalpy in both the structures as already has been given in Section 2. The variations of enthalpy for B1 and B2 phases of $\mathrm{AmBi}$ and $\mathrm{CfBi}$ have been plotted versus pressure in Fig. 3 and Fig. 4, respectively. The particular

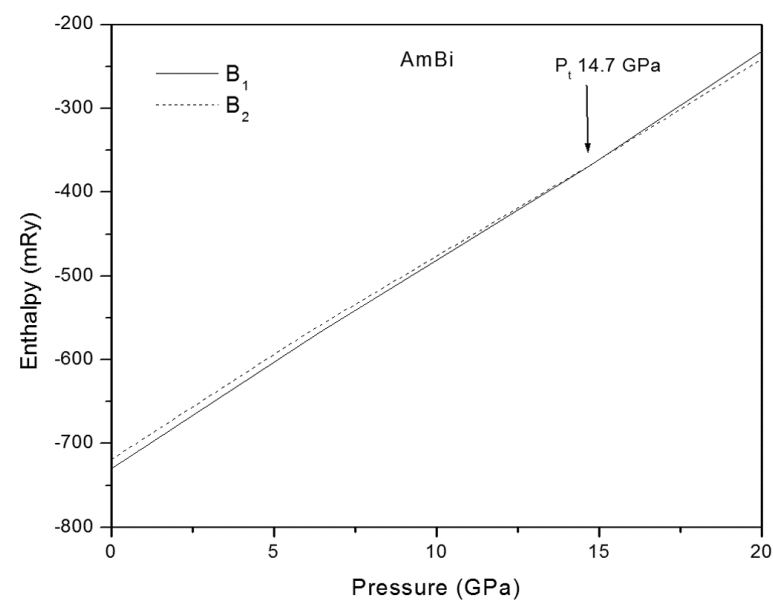

Fig. 3. Variation of enthalpies in B1 and B2 phases for AmBi.

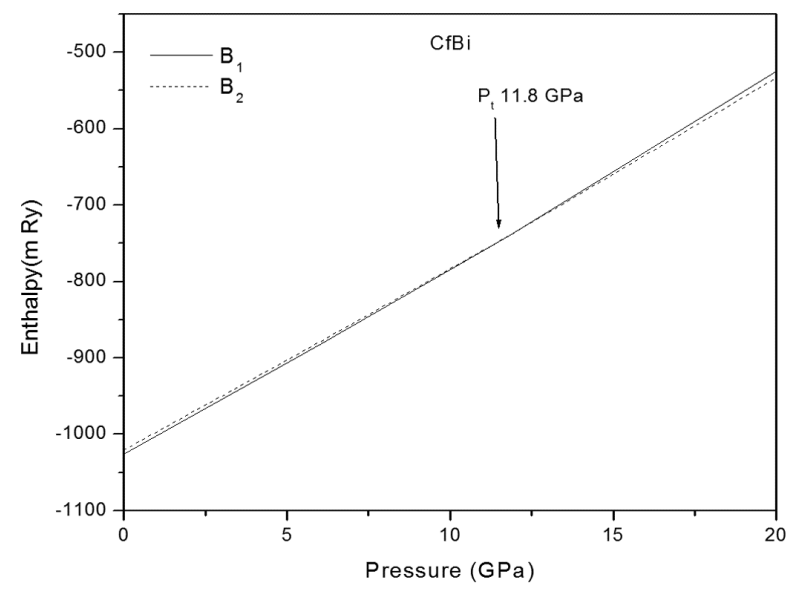

Fig. 4. Variation of enthalpies in B1 and B2 phases for CfBi.

pressure at which these two phases coexist is called the phase transition pressure. The calculated values of the phase transition pressure for the studied pnictides have been compared with theoretical [11, 12] and experimental [8, 10] results. The calculated values of bulk modulus for $\mathrm{AmBi}$ and CfBi are also presented in Table 1.

\subsection{Electronic properties}

The electronic band structures (BS) of AmBi have been shown in Fig. 5 and Fig. 6 for B1 and B2 phase respectively. It is obvious from this figure that the pnictide shows metallic behavior which 
Table 1. Calculated equilibrium lattice parameter a ( $)$, bulk modulus B (GPa), number of f-states at the Fermi level $\mathrm{N}_{\mathrm{f}}$ (states/Ry cell) and volume collapse of $\mathrm{AmBi}$ and $\mathrm{CmBi}$.

\begin{tabular}{|c|c|c|c|c|}
\hline \multirow[t]{2}{*}{ Properties } & \multicolumn{2}{|c|}{$\mathrm{AmBi}$} & \multicolumn{2}{|c|}{$\mathrm{CfBi}$} \\
\hline & $\mathrm{NaCl}$-type & CsCl-type & NaCl-type & CsCl-type \\
\hline \multicolumn{5}{|c|}{ Lattice parameter $(\AA ̊)$} \\
\hline Present & 6.1 & 3.5763 & 6.02 & 3.71 \\
\hline Exp. & $6.3[8]$ & & $6.4[8]$ & \\
\hline \multicolumn{5}{|c|}{ Bulk modulus (GPa) } \\
\hline Present & 75.45 & 58.38 & 102.22 & 66.62 \\
\hline Exp. & $75[10]$ & & $105[10]$ & \\
\hline \multicolumn{5}{|c|}{$\mathrm{N}_{\mathrm{f}}($ states/Ry cell $)$} \\
\hline Present & 730.18 & 730.52 & 1020.78 & 1024.91 \\
\hline \multicolumn{5}{|l|}{ Exp. } \\
\hline \multicolumn{5}{|c|}{$\begin{array}{l}\text { Structural phase transi- } \\
\text { tion pressure }(\mathrm{GPa})\end{array}$} \\
\hline Present & \multicolumn{2}{|c|}{14.7} & \multicolumn{2}{|c|}{11.8} \\
\hline Exp. & \multicolumn{2}{|c|}{$15[10]$} & \multicolumn{2}{|c|}{$11-12$ [10] } \\
\hline Others & \multicolumn{2}{|c|}{$16[11], 15.6[12]$} & \multicolumn{2}{|c|}{$11.2[11], 11.5[12]$} \\
\hline \multicolumn{5}{|l|}{$\mathrm{V} / \mathrm{V}_{0}$} \\
\hline Present & \multicolumn{2}{|c|}{11.2} & \multicolumn{2}{|c|}{6.5} \\
\hline Exp. & \multicolumn{2}{|c|}{12 [10] } & \multicolumn{2}{|c|}{$7[10]$} \\
\hline Others & \multicolumn{2}{|c|}{10 [11], 10.8 [12] } & \multicolumn{2}{|c|}{$11[11] 7.6[12]$} \\
\hline
\end{tabular}

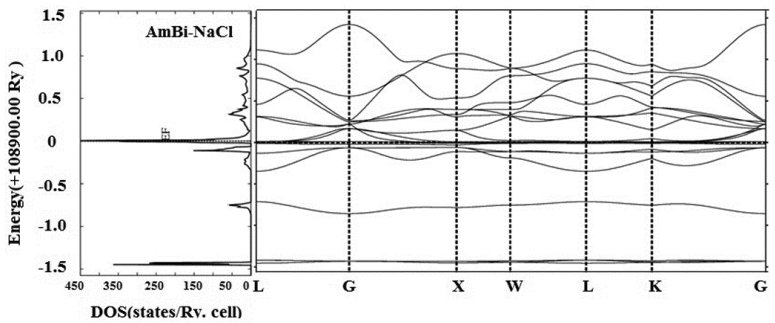

Fig. 5. Electronic band structure (BS) and total density of states of $\mathrm{AmBi}$ in $\mathrm{NaCl}$ phase.

is confirmed by the presence of f-electron at the Fermi level as reported in Table 1. To understand further the elementary contribution of all the atoms, the partial densities of states (PDOS) have been studied. These PDOS have been presented in Fig. 7 for AmBi. It is clearly shown from this figure that the lower valence band lying at $-1.45 \mathrm{Ry}$ is due to the Am-p states of the AmBi. The upper balance bands lying between -0.5 and $-1.0 \mathrm{Ry}$ are due to hybridization of Bi-s and Cf-d states. The

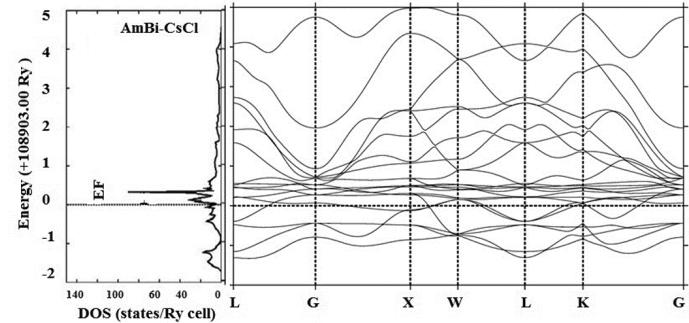

Fig. 6. Electronic band structure (BS) and total density of states of $\mathrm{AmBi}$ in $\mathrm{CsClCl}$ phase.

Am-d and Bi-p states are highly localized at the Fermi level.

Similar analysis has also been performed for $\mathrm{CfBi}$ which shows almost the same behavior. For $\mathrm{B} 1$ and $\mathrm{B} 2$ phases the electronic band structures of CfBi are shown in Fig. 8 and Fig. 9. As seen in these figures, some bands cross the Fermi level, which indicates the metallic behavior of this pnictide. The bands lie just above the Fermi level, indicating hybridization of f-sate. To confirm the 

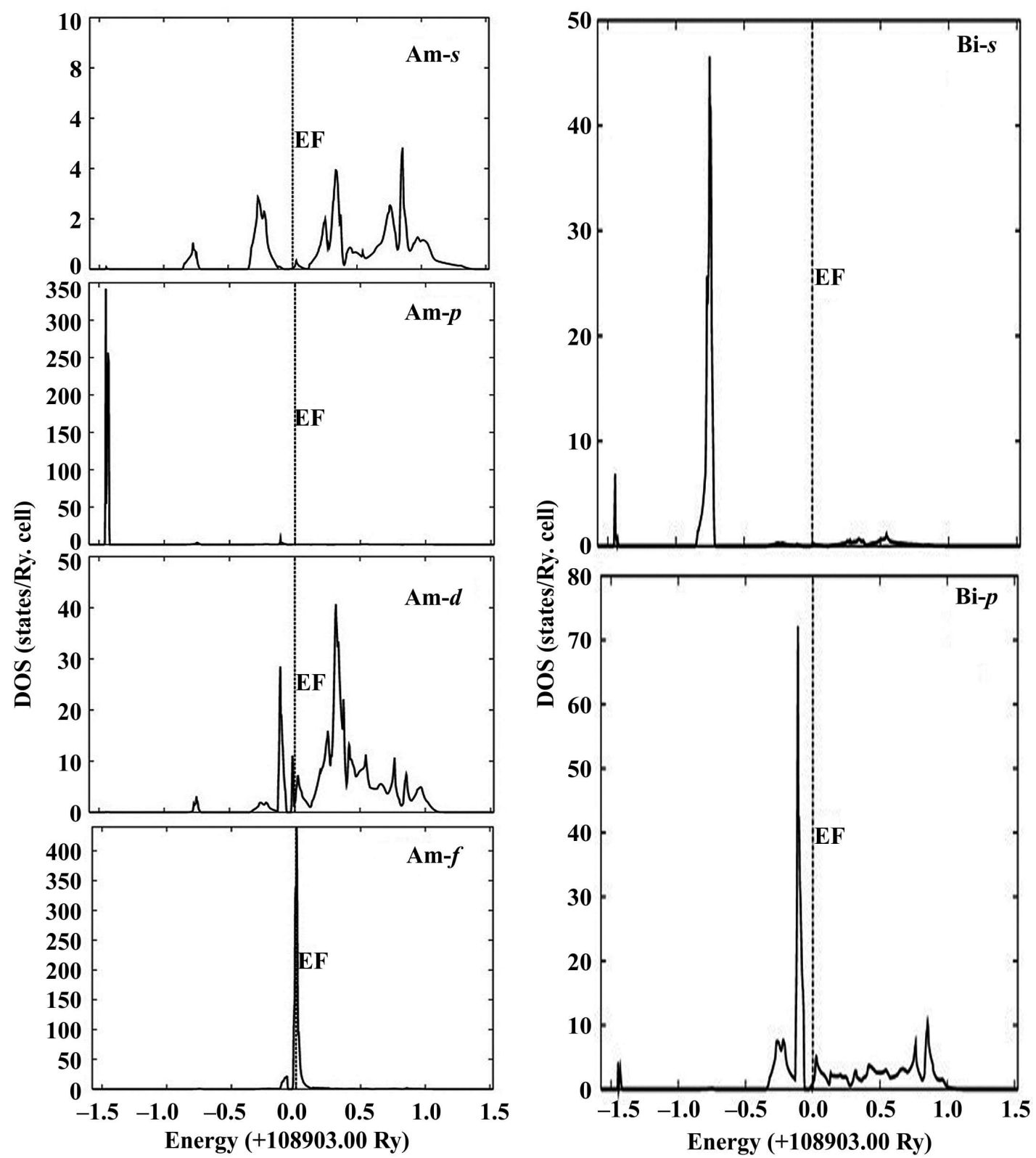

Fig. 7. Partial density of states (PDOS) in the $\mathrm{NaCl}$ and $\mathrm{CsCl}$ phase of $\mathrm{AmBi}$.

metallic behavior of this compound, PDOS have also been calculated for the B1 and B2 phase. It is clearly shown from Fig. 10 that the lower valence band lying around $-1.48 \mathrm{Ry}$ is due to the Cf-p states for CfBi. The upper balance band lying at $-1.0 \mathrm{Ry}$ is due to Bi-s state. At Fermi level Cf-f states are highly localized. Also Cf-d and Bi$\mathrm{p}$ states are hybridized at $\mathrm{G}$ point and $\mathrm{K}$ point, respectively.
As mentioned in section 3.1 these compounds undergo structural phase transition from $\mathrm{B} 1$ to $\mathrm{B} 2$ phase at 14.7 GPa for $\mathrm{AmBi}$ and 11.8 $\mathrm{GPa}$ for $\mathrm{CfBi}$. The electronic BS and DOS for the pnictides in B2 phase are shown in Fig. 8 to Fig. 10. These pnictides maintain their metallic properties at high pressure. The nature of the bands and DOS in B2 phase is almost the same as that of the B1 phase for these two compounds, with only 


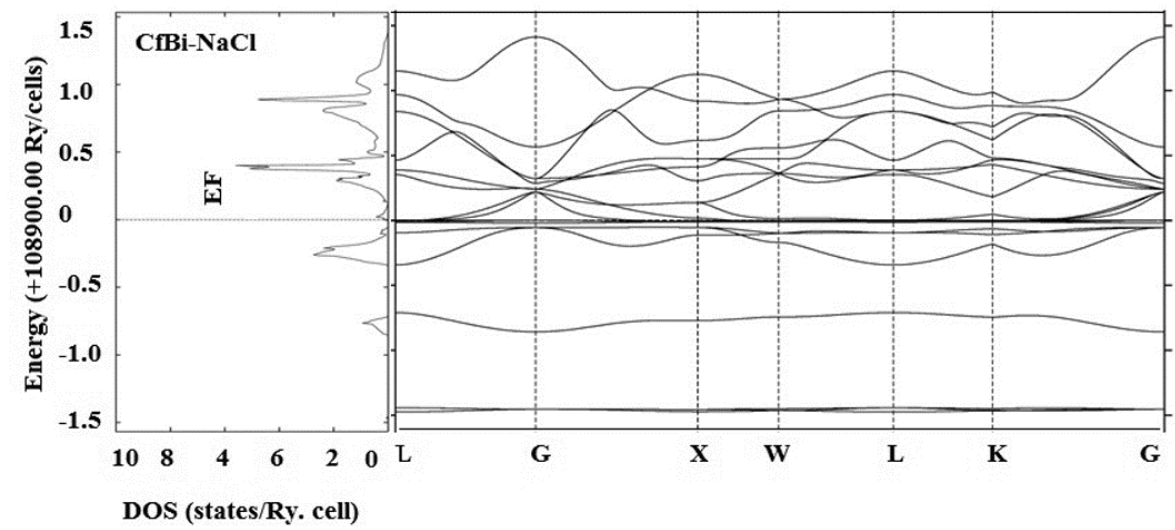

Fig. 8. Electronic band structure (BS) and total density of states of $\mathrm{CfBi}$ in $\mathrm{NaCl}$ phase.

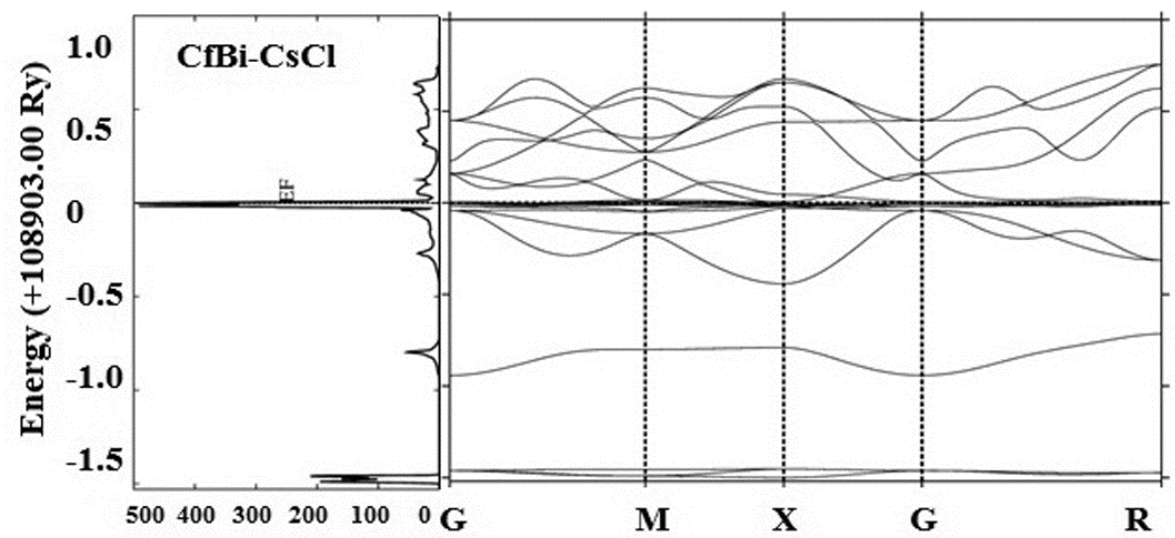

DOS (states/Ry cell)

Fig. 9. Electronic band structure (BS) and total density of states of $\mathrm{CfBi}$ in $\mathrm{CsCl}$ phase.

a minor difference. The lower energy bands are shifted to the higher energy bands near the Fermi level. So, the bands seem to be more compressed. Typical strongly correlated electron systems are found in transition metal compounds, where the Fermi level EF crosses $d$ bands. Rare earth compounds with f-electron bands crossing $\mathrm{EF}$ and organic conductors with $\mathrm{p}$ bands at $\mathrm{EF}$ are also well known correlated electron systems. A characteristic and common feature of the strongly correlated electron systems is that their bands crossing the Fermi level have narrow bandwidths. Nevertheless, the overall encouraging results for the Fermi surface obtained by LDA calculations provided obvious confidence that the Fermi surface is little affected by strong correlations in many materials [21].

\section{Conclusions}

The electronic and structural properties of rare earth pnictides ( $\mathrm{XBi} ; \mathrm{X}=\mathrm{Am}, \mathrm{Cf}$ ) have been using self-consistent tight binding linear muffin tin orbital (TB-LMTO) method within the local density approximation (LDA). At normal conditions, the studied pnictides are stable in the NaCl-type (B1-phase) structure and undergo a structural phase transition into CsCl-type (B2-structure). These compounds are found to be metallic in nature in both the phases because of large number 

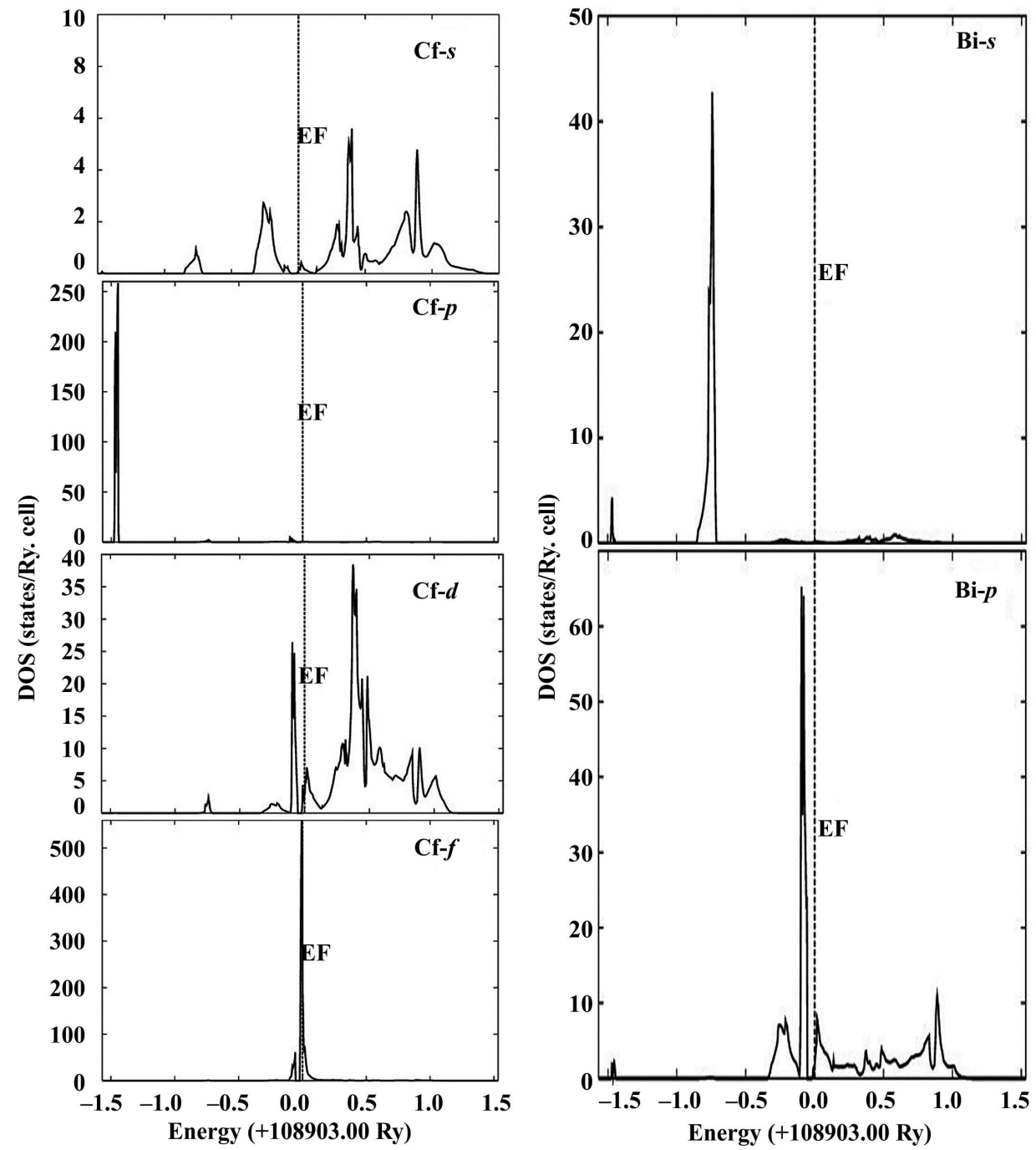

Fig. 10. Partial density of states (PDOS) in the $\mathrm{NaCl}$ and $\mathrm{CsCl}$ phase of $\mathrm{CfBi}$.

of f-electron states of pnictide ion present at the Fermi level. The calculated structural phase transition and volume collapse show good agreement with experimental $[8,10]$ and other theoretical results $[11,12]$.

\section{References}

[1] Leger J.M., Physica B, 190 (1993), 84.

[2] Gensini M., Haire R.G., Benedict U., HulLIGER F., Physica B, 190 (1993), 75.

[3] Leger J.M., Vedel I., Redon A.M., RossatMignod J., J. Magn. Magn. Mater., 63 - 64 (1987), 49.

[4] Svane A., Strange P., Temmerman W.M., Szotek Z., Winter H., Petit L., Phys. Status Solidi $B, 223$ (2001), 105 .
[5] Shirotani I., Hayashi J., Yamanashi K., Ishimatsu N., Shimomura O., Kikegawa T., Phys. Rev. $B, 64$ (2001), 132101.

[6] Shirotani I., Hayashi J., Yamanashi K., IshiMatsu N., Shimomura O., Kikegawa T., Solid State Commun., 125 (2003), 543.

[7] Johansson B., Phys. Rev. B, 11 (1975), 2836.

[8] Vogt O., Mattenberger K., J. Alloy. Compd., 223 (1995), 226.

[9] Yakovin I.N., Komesu T., Dowben P.A., Phys. Rev. B, 66 (2002), 035406.

[10] Benedict U., J. Alloy. Compd., 223 (1995), 216.

[11] Ahirwar A.K., AYNYAS M., Arya B.S., SANYAL S.P., J. Nucl. Mater., 413 (2011), 30.

[12] Bhardwaj P., Sarwan M., Dubey R., Singh S., J. Mol. Struct., 1043 (2013), 85.

[13] Christensen N.E., Phys. Rev. B, 32 (1985), 207.

[14] Andersen O.K., Phys. Rev. B., 12 (1975), 3060. 
[15] Andersen O.K., Jepsen O., Phys. Rev. Lett., 53 (1984), 2571.

[16] Kohn W., Sham L.J., Phys. Rev. A, 140 (1965), 1133.

[17] Barth Van U., Hedin L., J. Phys. C, 5 (1972), 1629.

[18] Srivastava V., Rajagopalan M., Sanyal S.P., $J$. Magn. Magn. Mater, 321 (2009), 607.

[19] Jepsen O., Andersen O.K., Solid State Commun., 9 (1971), 1763.
[20] BIRCH F., J. Geophys. Rev., 83 (1978), 1257.

[21] Imada M., MiYake T., J. Phys. Soc. Jpn., 20 (2010), 1.
Received 2015-03-27

Accepted 2015-12-19 